Präv Gesundheitsf 2021 · 16:16-20 https://doi.org/10.1007/s11553-020-00792-y Eingegangen: 24. März 2020

Angenommen: 1. Mai 2020

Online publiziert: 26. Mai 2020

(c) Der/die Autor(en) 2020

Natascha Mojtahedzadeh ${ }^{1}$ (D) - Felix Alexander Neumann ${ }^{2} \cdot$ Matthias Augustin $^{3}$. Birgit-Christiane Zyriax ${ }^{2}$. Volker Harth ${ }^{1}$. Stefanie Mache ${ }^{1}$

'Zentralinstitut für Arbeitsmedizin und Maritime Medizin (ZfAM), Universitätsklinikum HamburgEppendorf (UKE), Hamburg, Deutschland

${ }^{2}$ Institution: Präventivmedizin und Ernährung, Institut für Versorgungsforschung in der Dermatologie und bei Pflegeberufen (IVDP), Universitätsklinikum Hamburg-Eppendorf (UKE), Hamburg, Deutschland

${ }^{3}$ Institut für Versorgungsforschung in der Dermatologie und bei Pflegeberufen (IVDP),

Universitätsklinikum Hamburg-Eppendorf (UKE), Hamburg, Deutschland

\title{
Das Gesundheitsverhalten von Pflegekräften - aktueller Forschungsstand, Potenziale und mögliche Herausforderungen
}

anspruchungsfolgen, u.a. durch soziale Konflikte mit Klient*innen, ist somit für diese Beschäftigtengruppe erhöht [22, 38, 41]. Hinsichtlich der steigenden Anzahl an ambulanten Pflegediensten in Deutschland (2017 zu 2015: +700 ambulante Pflegeeinrichtungen; [37]) treten die Beschäftigten der ambulanten Pflege und ihre Gesundheit zunehmend in den Vordergrund. In Bezug auf die hohe Krankheitsquote in der Pflege liegt großes Präventionspotenzial in gesunden Arbeitsbedingungen sowie in der Stärkung des Gesundheitsverhaltens der Pflegekräfte.

Während erste Ergebnisse aus der Untersuchung beanspruchender Arbeitsbedingungen aus der stationären Pflege Deutschlands existieren [42], fehlen hingegen fundierte wissenschaftliche Erkenntnisse zum Gesundheitsverhalten ambulant tätiger Pflegekräfte. Die Tätigkeit in der ambulanten Pflege bedeutet oftmals Hektik bzw. Stress durch Termindruck und Beschleunigung in der Arbeitswelt [7], die Wahl und die Durchführung von gesunden Verhaltensweisen scheint erschwert [21]. Der vorliegende Artikel soll eine Übersicht zum aktuellen Forschungsstand des Gesundheitsverhaltens von Pflegekräften, insbesondere aus der ambulanten Pflege, darstellen, mögliche Potenziale beleuchten und praktische Herausforderungen aufdecken. Das Gesundheitsverhalten deutscher Pflegekräfte wurde bisher nicht untersucht.

\section{Gesundheitsverhalten}

Mögliche negative Beanspruchungsfolgen (u.a. das Stresserleben) können zu gesundheitlichen Beeinträchtigungen führen [33]. In diesem Kontext wird das Gesundheitsverhalten relevant. Gewohnheitsmäßige Handlungsweisen, die den individuellen Gesundheitszustand erhalten oder fördern, gehören unabdingbar in den Bereich von Erholungsund Entspannungseinheiten [3]. In diesem Zuge wird zwischen positiven (z.B. Förderung persönlicher Weiterentwicklung, gesunde Ernährung) und risikoreichen Verhaltensweisen (z.B. Tabak-/ Alkoholkonsum, Bewegungsmangel) differenziert [1, 6]. Aus den Ergebnissen der DEGS1-Studie geht hervor, dass $16,6 \%(n=1405)$ der deutschen Bürger*innen in den letzten 12 Monaten mindestens eine verhaltenspräventive Intervention in Anspruch genommen haben, wobei Frauen den Fokus auf Entspannung legten, Männer sich eher mit Bewegung befassten [23]. Zum gegenwärtigen Zeitpunkt existieren kaum Erkenntnisse zu einzelnen Gesundheitsverhaltensweisen von Beschäftigten aus der ambulanten Pflege. Aktuelle em- 
pirische Befunde aus dem Bereich der Altenpflege liefern lediglich Wirth et al. [45]. Im Vergleich zu stationär tätigen Pfleger ${ }^{\star}$ innen und Erzieher ${ }^{*}$ innen zeigten diese die höchste Raucherquote auf (55\% zu 12\% bzw. 4\%; [45]) und liegen somit über dem Durchschnitt der deutschen Gesamtbevölkerung [48]. Auch konnten eher ungesunde Essgewohnheiten festgestellt werden [45]. Stetige Schichten zeigten in der stationären Pflege die Begünstigung gesundheitsförderlicher Verhaltensweisen [29].

\section{Aktueller Forschungsstand}

Positive und negative Gesundheitsverhaltensweisen werden im vorliegenden Artikel in den Bereichen Ernährungsverhalten, körperliche Aktivität, Rauchund Pausen-/Regenerationsverhalten unterteilt. Generell liegen aus dem Pflegesetting nur wenige Studien vor, die mehrheitlich ihren Schwerpunkt auf den stationären Kontext setzen.

\section{Ernährungsverhalten}

Das Ernährungsverhalten beschreibt die Auswahl von Lebensmitteln, Motiven und Ernährungsweisen, die in ernährungsbedingten Problematiken, wie beispielsweise Adipositas oder Essstörungen, münden können [25].

Aus der stationären Pflege sind ungesunde Ernährungsgewohnheiten (zuckerhaltige Lebensmittel) bereits bekannt. Das mobile Setting in der ambulanten Pflege, welches möglicherweise fehlende Kantinen aufweist, könnte die Inanspruchnahme regelmäßiger Pausen erschweren. Dies deutet auf potenzielle Hindernisse hinsichtlich einer regelmäßigen Mahlzeiteneinnahme und gesunder Ernährung hin, wodurch der Verzehr (ungesünderer) Zwischenmahlzeiten $\mathrm{zu}$ erwarten wäre [18]. Erlebter Arbeitsstress wurde in der stationären Pflege mit höherer Kalorienzufuhr assoziiert [47]. Das Mehrschichtsystem (Früh-, Spät- und Nachtdienst) führte zur geringsten Energiezufuhr im Spätdienst. Die meisten Kohlenhydrate wurden in der Nachtschicht bei gleichzeitig höchstem Stresserleben und Fett gehäuft in der Frühschicht verzehrt [20]. Wirth et al. [45] heben die Auswirkungen ungünstiger Ernährungsweisen bei Altenpfleger*innen hervor. So zeigen diese bereits in der Ausbildung adipöse Anzeichen auf. Die Arbeitsanforderungen im Schichtsystem erschweren überdies eine ausreichende Flüssigkeitszufuhr laut einer Befragung aus der stationären Pflege [17]. Ergebnisse aus der Forschung aus der stationären Pflege unter zusätzlichem Einbezug von Hebammen zeigen allerdings eine erhöhte Koffeinzufuhr [16]. Über das Trinkverhalten von ambulant tätigen Pflegekräften sind bisher keine wissenschaftlichen Erkenntnisse verfügbar.

\section{Körperliche Aktivität}

Körperliche Aktivität wird als jegliche Bewegung des Körpers beschrieben, die von Muskeln ausgeht und dabei Energie verbraucht. Ein Bewegungsmangel stellt einen Risikofaktor für die Gesundheit dar und kann u. a. Bluthochdruck sowie koronare Herzerkrankungen begünstigen [46]. Wechselnde Nachtschichten haben sich sowohl in der stationären Pflege als auch bei Hebammen als reduzierenden Faktor körperlicher Aktivität in der Freizeit erwiesen [31]. Bestehende belastende Arbeitsbedingungen wie die Arbeitsumgebung oder Schichtarbeit seien ebenfalls mögliche Gründe für eine geringe körperliche Aktivität und daraus resultierendem Übergewicht [13]. Im Vergleich zu anderen Krankenhausberufsgruppen zeigen Pfleger*innen die geringste körperliche Aktivität auf [14]. Geringe körperliche Aktivität $(<2 \mathrm{~h})$ ist in der Freizeit charakterisiert in Deutschland bereits in der Ausbildung in der Alten- und Krankenpflege [45]. Aus der ambulanten Pflege sind bisher keine wissenschaftlichen Erhebungen in Bezug auf die körperliche Aktivität der Beschäftigten verfügbar.

\section{Rauchverhalten}

Rauchen als ein Beispiel für negatives Gesundheitsverhalten ist in Deutschland stark verbreitet. Auf Grundlage der Datenbasis der GEDA 2014/2015-EHISStudie („Gesundheit in Deutschland aktuell“) haben jedoch zum Zeitpunkt der Befragung 26,6\% der Frauen und 35,0\% der Männer das Rauchen eingestellt [48]. Den Blick auf die Beschäftigten der stationären Pflege gerichtet, wird deutlich, dass ein hoher Tabakkonsum auf arbeitsplatzbezogenes Stressempfinden zurückzuführen sein kann: Die wöchentliche Arbeitszeit, die als negativ empfunden wird (z. B. Lage, Länge), ist mit vermehrtem Rauchverhalten wie auch Schlafmangel assoziiert [19, 36]. Auch in der Ausbildung zeigen lernende Pfleger*innen bereits regelmäßigen $\mathrm{Ni}$ kotinkonsum [26]. Zum Rauchverhalten von deutschen ambulant beschäftigten Pflegekräften existieren bisher jedoch keine fundierten Erkenntnisse.

\section{Pausen- und Regenerations- verhalten}

Um negative Beanspruchungsfolgen $\mathrm{zu}$ vermeiden, sollten regelmäßige Pausen eingehalten werden [15]. Erholungspausen während der Arbeitszeit haben in der Forschung eindeutige gesundheits- und leistungsförderliche Effekte gezeigt: Sie fördern Produktivität, verringern Ermüdungserscheinungen sowie Disstress und erhalten damit die Arbeitssicherheit der Beschäftigten [5, 40]. In der Literatur wird vermehrt auf die ungenügende Pausenumsetzung in der Praxis unter Krankenpfleger*innen verwiesen. Häufig geschieht dies durch den Verzug der Pausen oder Unterbrechungen während der Pausenzeit [43]. Auf der Datenbasis der Erwerbstätigenbefragung durch das Bundesinstitut für Berufsbildung (BIBB) in Kooperation mit der Bundesanstalt für Arbeitsschutz und Arbeitsmedizin (BAuA) BIBB/BAuA-Erwerbstätigenbefragung kommt es unter der Berufsgruppe der Pfleger ${ }^{*}$ innen (darunter stationäre und ambulante Pfleger ${ }^{\star}$ innen, $n=746$ ) zu erhöhtem Pausenausfall durch Mehrarbeit, Zeitdruck sowie Schichtarbeit. Darüber hinaus wird von Störungen/ Unterbrechungen und mengenmäßiger Überforderung durch zu hohe Arbeitsdichte während der Arbeitszeit berichtet [10], was belastend für die Beschäftigten sein kann [2]. Da ambulante Pflegekräfte ihre Arbeitszeit während der Fahrt und bei den Pflegekräften zu Hause verbringen, sei an dieser Stelle 
zu evaluieren, inwiefern sich deutsche Pflegekräfte während der Arbeitszeit im ambulanten Setting erholen können. Eine besondere Herausforderung stellen die tätigkeitsimmanenten Bedingungen der Arbeitsorganisation dar. Zeit- und Leistungsdruck, denen die Pflegekräfte ausgesetzt sind, erschweren die Einhaltung gesetzlich festgelegter Pausen. Ein Pausenausfall kann als „zeitsparendes Bewältigungsverhalten “ interpretiert werden [27]. Auffällig ist die scheinbar aktiver wahrgenommene Pauseneinhaltung von Raucher*innen im Vergleich zu Nicht-Raucher*innen unter stationären Pflegekräften: Die Tabaksucht wurde durch regelmäßige Pausen befriedigt [34]. Unterbesetzung hat sich in der Pflege ebenfalls als begünstigender Pausenausfallfaktor gezeigt [44].

Unter Regeneration wird eine nichtarbeitsplatzbezogene Erfahrung beschrieben, die dem Wohlbefinden der Individuen sowie der beruflichen Leistung dient [4]. Als Regenerationstechniken wurden in einer Befragung von auszubildenden Pflegekräften aus Amerika z.B. Yoga oder Meditationen genannt. Diese werden allerdings eher gelegentlich angewendet [32]. Dazu zählt laut einer Befragung walisischer Pflegekräfte ebenfalls Zeit, sich während der Arbeitszeit zurückzuziehen [12].

Die Arbeit im Schichtdienst sowie Arbeitsanforderungen haben sich in der stationären Pflege bereits als beeinträchtigend hinsichtlich der Schlafqualität und der Durchführung positiver Gesundheitsverhaltensweisen (körperliche Aktivität, ausreichende Regeneration) erwiesen [19, 28]. Ein kurzer Erholungsschlaf während der Arbeitszeit hat eine bessere Erholung nach der Schicht zeigen können [30]. Allerdings ist bis dato das Pausen- und Regenerationsverhalten von deutschen Pflegekräften nur wenig untersucht worden.

\section{Zusammenfassung und Ausblick}

Aus der vorliegenden Übersicht des aktuellen Forschungsstands geht hervor, dass gegenwärtig kaum Erkenntnisse vom Gesundheitsverhalten von deutschen Pfleger ${ }^{*}$ innen vorliegen. Fundierte empirische Studien bezüglich der Analyse

Präv Gesundheitsf 2021 · 16:16-20 https://doi.org/10.1007/s11553-020-00792-y

(c) Der/die Autor(en) 2020

N. Mojtahedzadeh · F. A. Neumann · M. Augustin · B.-C. Zyriax · V. Harth · S. Mache

Das Gesundheitsverhalten von Pflegekräften - aktueller Forschungsstand, Potenziale und mögliche Herausforderungen

\section{Zusammenfassung}

Der Artikel bietet eine Übersicht zum aktuellen Forschungsstand bezüglich des Gesundheitsverhaltens von Beschäftigten aus der Pflegebranche. Trotz der wachsenden Anzahl an ambulant gepflegten Personen und damit dem Beschäftigtenwachstum in dieser Branche existieren aktuell kaum Erkenntnisse und empirische Befunde über das Gesundheitsverhalten von deutschen Pflegekräften. Die bisher verfügbare Informationslage deutet allerdings auf eine ungesunde Ernährungsweise, mangelhafte körperliche Aktivität sowie auf mangelnde Möglichkeiten von Pausen- und Regenerationseinheiten hin. Forschungsund Interventionsarbeiten zur Analyse des Arbeits- und Gesundheitsverhaltens als Grundlage zur Entwicklung bedarfsgerechter gesundheitsförderlicher Maßnahmen sind unabdingbar.

Schlüsselwörter

Betriebliche Gesundheitsförderung · Deutsche Pflegekräfte · Gesunde Verhaltensweisen . Pflege $\cdot$ Regeneration

\section{The health behaviour of caregivers-current state of research, potentials and challenges}

\section{Abstract}

This article aims to contribute to the current state of research regarding health behaviour among employees in the healthcare sector. The number of people in need of care is continually growing and thus there is a growing need of employees in this field. Nevertheless little is known about German caregivers' health behaviour. There are some indications that caregivers show poor eating habits, have insufficient physical activity and lack opportunities for breaks or regeneration periods. Therefore future research and interventions analysing caregivers' work and health behaviour are needed as a basis for development of future needs-based health promotion interventions.

Keywords

Care - German caregivers - Health promotion . Healthy behavioural pattern $\cdot$ Regeneration dieser Verhaltensweisen fehlen gänzlich. Insbesondere auf nationaler Ebene ist die Forschungslage $\mathrm{zu}$ den ambulant tätigen Pflegekräften fehlend. Die Studie von Wirth et al. [45] zeigt Unterschiede im Gesundheitsverhalten zwischen Altenpfleger*innen und stationären Krankenpfleger*innen. Diese könnten sich möglicherweise durch die Mobilität der Altenpfleger*innen ergeben und sollten künftig näher untersucht werden.

Welche und inwiefern gesundheitsfördernde und regenerative bzw. negative Verhaltensweisen von Beschäftigten aus der ambulanten Pflege eingesetzt (oder unterlassen) werden, bleibt nichtsdestotrotz fraglich. Die vorhandene wissenschaftliche Literatur weist jedoch darauf hin, dass Pausen-/Regenerationsmuster sowie Ernährungs-, Bewegungs- und
Rauchverhalten sich als aufschlussreiche Parameter zur Untersuchung vom Gesundheitsverhalten von Pflegekräften erwiesen haben. Die ambulante Pflege bringt unterschiedliche negative Belastungen mit sich. Das mobile Setting birgt eine ständig wechselnde Arbeitsumgebung. Staus und schlechte Witterungsverhältnisse können zusätzlich zur übermäßigen Arbeitsmenge belastend sein und zum Zeitverzug des Zeitplans der Beschäftigten führen [11]. Im Setting der ambulanten Pflege könnten Rückzugseinheiten durch fehlende Raumgestaltungsmöglichkeiten sowie der zu verbringenden Zeit bei Patient*innen und im Verkehrsmittel auf dem Weg dorthin, generell erschwert sein. Fehlende Pauseneinheiten und -räume sowie die geforderte Arbeitszeit 
unterwegs im Verkehr könnten überdies die Ernährungsweise der ambulant tätigen Beschäftigten negativ beeinflussen. Die mangelnde Pausenzeit beim Transfer zwischen den Patient*innen [11] könnte den Rückgriff auf ungesunde Snacks begünstigen. Die speziellen Arbeitsanforderungen (Mobilität, Zeitdruck etc.; [11]) aus der ambulanten Pflege stellen einen Risikofaktor für einen übermäßigen Koffeinkonsum dar. Arbeitsbedingungen, wie eine enge Schichtplanung sowie fehlende feste Örtlichkeiten, begünstigen möglicherweise eine eingeschränkte Flüssigkeitszufuhr.

Die arbeitsbezogenen Belastungen (hohe Arbeitsdichte, keine Regenerationsmöglichkeit) könnten Auswirkungen auf die Motivation der körperlichen Aktivität nach der Arbeitsschicht mit sich bringen. Die bekannte Literatur, welche auf Pausenausfall und starkem Rauchverhalten in der Pflege hindeutet [10, 19], könnte hinsichtlich der Beschäftigten aus der ambulanten Pflege relevant werden und fehlende Regenerationseinheiten implizieren. Insbesondere im Hinblick auf die steigende Anzahl Pflegebedürftiger sowie der erhöhten Inanspruchnahme ambulanter Pflegeleistungen in Deutschland [37] rücken die Beschäftigen aus der ambulanten Pflege (auch hinsichtlich der hohen Krankenstände) und somit ihre Gesundheit in den Vordergrund. Negative arbeitsorganisatorische Bedingungen in der Pflegebranche [11] können das Gesundheitsverhalten der Beschäftigten beeinflussen. Durch die Zunahme an ambulanten Pflegediensten (= mehr Beschäftigte in der ambulanten Pflegebranche) und der Tatsache, dass die Gesundheit bzw. das Gesundheitsverhalten der ambulanten Pflegekräfte in Deutschland nicht untersucht wurde, stellt sich das Forschungsdesiderat heraus. Eine umfassende Analyse dessen kann die Basis für eine bedarfsgerechte, zielgruppenspezifische Gestaltung gesundheitsförderlicher Maßnahmen auf Verhaltens- und Verhältnisebene im Sinne der BGF bilden. Darauf aufbauend können Interventionsstudien die Wirkung auf das Gesundheitsverhalten der deutschen Pflegekräfte untersuchen. Im Fokus dieses Forschungsinteresses sollten dabei gesunde Arbeitsbedin- gungen sowie Implikationen für das Gesundheitsverhalten liegen. So können Gesundheit, Motivation und Produktivität der Beschäftigten langfristig sichergestellt werden, was ferner im wirtschaftlichen Interesse der Betriebe liegt. Zukünftige praktische Herausforderungen werden in der Arbeitsorganisation gesehen. Die Verantwortung der Gesundheit der Beschäftigten obliegt ihren Arbeitgeber*innen und Führungskräften. Diese bedürfen mehr Kenntnisse und Aufklärung über mögliche Auswirkungen arbeitsplatzbezogener Belastungen auf das Gesundheitsverhalten ihrer Mitarbeiter*innen. Die Implementation eines BGM auf Führungsebene, in dessen Verantwortung die BGF steht [9], erreicht für die Pflegebranche zunehmend Relevanz. So kann nicht nur das Gesundheitsverhalten der Beschäftigten gefördert werden. Arbeitsunfähigkeitstagen und Frühverrentungen wird so langfristig entgegengewirkt.

\section{Fazit für die Praxis}

- Führungskräfte aus dem Bereich der ambulanten Pflege sollten die Gesundheit ihrer Mitarbeiter*innen verstärkt in den Vordergrund stellen.

- Maßnahmen zur Förderung positiver Gesundheitsverhaltensweisen auf Verhältnis-/Verhaltensebene sollten zum Erhalt der Gesundheit, Motivation und Produktivität der Beschäftigten in der ambulanten Pflege eingeleitet und auf ihre Wirksamkeit überprüft werden.

- Die Implementation eines betrieblichen Gesundheitsmanagements, mit dem Fokus fortlaufend Maßnahmen im Sinne der betrieblichen Gesundheitsförderung zur Verbesserung der Gesundheit von ambulant tätigen Pfleger*innen zu entwickeln, wird angeraten.

\section{Korrespondenzadresse}

Natascha Mojtahedzadeh, M.A.

Zentralinstitut für Arbeitsmedizin und

Maritime Medizin (ZfAM), Universitätsklinikum Hamburg-Eppendorf (UKE)

Seewartenstraße 10| Haus 1 |, 20459 Hamburg, Deutschland

n.mojtahedzadeh@uke.de

Funding. Das Projekt wird von der Berufsgenossenschaft für Gesundheitsdienst und Wohlfahrtspflege (BGW) gefördert. Open Access funding provided by Projekt DEAL.

\section{Einhaltung ethischer Richtlinien}

Interessenkonflikt. N. Mojtahedzadeh, F.A. Neumann, M. Augustin, B.-C. Zyriax, S. Mache und V. Harth geben an, dass kein Interessenkonflikt besteht.

Für diesen Beitrag wurden von den Autoren keine Studien an Menschen oder Tieren durchgeführt. Für die aufgeführten Studien gelten die jeweils dort angegebenen ethischen Richtlinien.

Open Access. Dieser Artikel wird unter der Creative Commons Namensnennung 4.0 International Lizenz veröffentlicht, welche die Nutzung, Vervielfältigung, Bearbeitung, Verbreitung und Wiedergabe in jeglichem Medium und Format erlaubt, sofern Sie den/die ursprünglichen Autor(en) und die Quelle ordnungsgemäß nennen, einen Link zur Creative Commons Lizenz beifügen und angeben, ob Änderungen vorgenommen wurden.

Die in diesem Artikel enthaltenen Bilder und sonstiges Drittmaterial unterliegen ebenfalls der genannten Creative Commons Lizenz, sofern sich aus der Abbildungslegende nichts anderes ergibt. Sofern das betreffende Material nicht unter der genannten Creative Commons Lizenz steht und die betreffende Handlung nicht nach gesetzlichen Vorschriften erlaubt ist, ist für die oben aufgeführten Weiterverwendungen des Materials die Einwilligung des jeweiligen Rechteinhabers einzuholen.

Weitere Details zur Lizenz entnehmen Sie bitte der Lizenzinformation auf http://creativecommons.org/ licenses/by/4.0/deed.de.

\section{Literatur}

1. Anderson R (1984) Gesundheitsförderung: Ein Überblick. Europäische Monographien zur Forschung. Gesundheitserziehung 6:1-140

2. Baethge A, Rigotti T (2013) Auswirkung von Arbeitsunterbrechungen und Multitasking auf Leistungsfähigkeit und Gesundheit - Eine Tagebuchstudie bei Gesundheits- und Krankenpflegerlnnen. Bonifatius, Paderborn

3. Becker P (1992) Die Bedeutung integrativer Modelle von Gesundheit und Krankheit für die Prävention und Gesundheitsförderung.In:Paulus $P$ (Hrsg) Prävention und Gesundheitsförderung. Perspektiven für die psychosoziale Praxis. GwG, Köln, S91-108 
4. Binnewies C, Sonnentag S, Mojza EJ (2009) Feeling recovered and thinking about the good sides of one's work. J Occup Health Psychol 14:243-256

5. Blasche G, Pasalic S, Bauböck V-M et al (2017) Effects of rest-break intention on rest-break frequency and work-related fatigue. Hum Factors 59:289-298

6. Blättner B, Waller H (2018) Gesundheitswissenschaft. Eine Einführung in Grundlagen, Theorie und Anwendung. Kohlhammer, Stuttgart

7. Bleses $P$, Jahns $K$ (2016) Soziale Innovationen in der ambulanten Pflege. In: Becke $G$, Bleses $P$ Freirichs F, Goldmann M, Hinding B, Schweer MKW (Hrsg) Zusammen - Arbeit - Gestalten. Soziale Innovationen in sozialen und gesundheitsbezogenen Dienstleistungen. Springer VS, Wiesbaden, S127-144

8. Böhle F (2010) Neue Anforderungen an die Arbeitswelt - neue Anforderungen an das Subjekt. In: Keupp H, Dill H (Hrsg) Erschöpfende Arbeit. Gesundheit und Prävention in der flexiblen Arbeitswelt. transcript, Bielefeld, S77-96

9. Brandenburg U, Nieder P (2009) Betriebliches Fehlzeiten-Management. Instrumente und Praxisbeispiele für erfolgreiches Anwesenheits- und Vertrauensmanagement.Gabler, Wiesbaden

10. Bundesanstalt für Arbeitsschutz und Arbeitsmedizin (2014) BIBB/BAuA-2012. Factsheet 10. Arbeit in der Pflege-Arbeit am Limit? Arbeitsbedingungen in der Pflegebranche. https://www.baua.de/DE/ Angebote/Publikationen/Fakten/BIBB-BAuA-10. pdf? _ blob=publicationFile\& $v=4$. Zugegriffen: 18. Mai 2020

11. Bundesministerium Für Gesundheit (2017) Gesundheitsförderung für Pflegekräfte: Wer pflegt die Pflege? Ausgangslage: Die Arbeitssituation in derPflege. Praxisseiten Pflege 06/2017:X.0.1

12. Burnard $P$, Edwards D, Fothergill $A$ et al (2000) Community mental health nurses in Wales: self-reported stressors and coping strategies. J Psychiatr Ment Health Nurs 7:523-528

13. Chin AL, Nam S, LeeS-J (2016) Occupational factors associated with obesity and leisure-time physical activity among nurses: a cross sectional study. Int $J$ Nurs Stud 57:60-69

14. Chiou ST, Chiang JH, Huang N et al (2014) Health behaviors and participation in health promotion activities among hospital staff: which occupational group performs better? BMC Health Serv Res 14:474

15. Deutsches Institut Für Normung (2000) DIN EN ISO 10075-2:2000 Ergonomische Grundlagen bezüglich psychischer Arbeitsbelastung. Teil 2: Gestaltungsgrundsätze. Beuth, Berlin

16. Dorrian J, Paterson J, Dawson D et al (2017) Sleep, stress and compensatory behaviours in Australian nurses and midwives. Rev Saude Publica 45:922-930

17. Gifkins J, Jophnston A, Loudoun R (2018) The impact of shift work on eating patterns and self-care strategies utilised by experienced and inexperienced nurses. Chronobiol Int 35:811-820

18. Gupta CC, Coates AM, Dorrian J et al (2019) The factors influencing the eating behaviour of shiftworkers: what, when, where and why. Ind Health 57:419-453

19. Han K, TrinkoffAM, Storr CLet al (2012) Comparison of job stress and obesity in nurses with favorable and unfavorable work schedules. J Occup Environ Med 54:928-932

20. Heath G, Dorrian J, Coates A (2019) Associations between shift type, sleep, mood, and diet in a group of shift working nurses. Scand J Work Environ Health 45:402-412
21. Hurley S, Edwards J, Cupp J et al (2018) Nurses' perceptions of self as role models of health. West $J$ Nurs Res 40:1131-1147

22. Institut für Gesundheits- Und Sozialforschung GmbH (2006) DAK-BGW-Gesundheitsreport. Ambulante Pflege. Arbeitsbedingungen und Gesundheit in ambulanten Pflegediensten. DAK, Hamburg

23. Jordan S, Von Der Lippe E (2013) Teilnahme an verhaltenspräventiven Maßnahmen. Ergebnisse der Studie zur Gesundheit Erwachsener in Deutschland (DEGS1). Bundesgesundhbl 56:878-884

24. Kratzer N, Dunkel W (2011) Arbeit und Gesundheit im Konflikt.ZurEinführung. In:KratzerN, DunkelW, Becker K, Hinrichs S (Hrsg) Arbeit und Gesundheit im Konflikt. Analysen und Ansätze für ein partizipatives Gesundheitsmanagement. edition sigma, Berlin

25. Lacaille L (2013) Eating behavior. In: Gellman MD, Turner JR (Hrsg) Encyclopedia of behavioral medicine. Springer, New York, S641-642

26. Lehmann F, Von Lindeman K, Klewer J et al (2014) BMI, physical inactivity, cigarette and alcohol consumption in female nursing students: a 5-year comparison. BMCMed Educ 14:82

27. Lohmann-Haislah A, Wendsche J, Schulz A et al (2019) Einflussfaktoren und Folgen des Ausfalls gesetzlicher Ruhepausen bei Pflegekräften in Deutschland.ZArbeitswiss 73:418-438

28. Mcdowall K, Murphy E, Anderson K (2017) The impact of shiftworkon sleepqualityamong nurses. Occup Med 67:621-625

29. Najaf-AbadiHM, RezaeiB (2018) Health-promoting behaviours of Iranian nurses and its relationship with some occupational factors: a cross sectional study. J Nurs Manag 26:717-725

30. Palermo TA, Rotenberg L, Zeitoune RC et al (2015) Napping during the night shift and recovery after work among hospital nurses. Rev Lat Am Enferm 23:114-121

31. Peplonska B, Bukowska A, Sobala W (2014) Rotating night shift work and physical activity of nurses and midwives in the cross-sectional study in Łódź, Poland. Chronobiol Int 31:1152-1159

32. Petch-Levine D, Younr Cureton V, Canham D et al (2003) Health Practices of School Nurses. J Sch Nurs 19:273-280

33. Richter P, Hacker W (2012) Belastung und Beanspruchung. Stress, Ermüdung und Burnout im Arbeitsleben. Asanger, Kröning

34. Sarna L, Aguinaga Bialous S, Wells MJ et al (2009) Do you need to smoke to get a break?: smoking status and missed work breaks among staff nurses. Am JPrev Med 37:165-171

35. Schulz E (2012) Pflegemarkt: Drohendem Arbeitskräftemangel kann entgegengewirkt werden. DIW Wochenber 79:3-18

36. Silva-Costa A, Harter Griep R, Fischer FM et al (2012) Need for recovery from work and sleep-related complaints among nursing professionals. Work 41:3726-3731

37. Statistisches Bundesamt (2018) Pflegestatistik - Pflege im Rahmen der Pflegeversicherung: Deutschlandergebnisse

38. Suadicani P, Olesen K, Bonde JP et al (2014) Psychosocial work conditions associated with sickness absence among hospital employees. Occup Med 64:503-508

39. Techniker Krankenkasse (2019) Gesundheitsreport. Pflegefall Pflegebranche? So geht's Deutschlands Pflegekräften. Techniker Krankenkasse, Hamburg
40. Tucker $P$ (2003) The impact of rest breaks upon accident risk, fatigue and performance: a review. WorkStress 17:123-137

41. VoltmerE, WingenfeldK, Spahn Cetal(2013)Workrelated behaviour and experience patterns of nurses in different professional stages and settings compared to physicians in Germany. Int J Mental Health Nurs 22:180-189

42. Vu-Eickmann P, Loerbroks A (2017) Psychosocial working conditions of physician assistants: results from a qualitative study on occupational stress, resources, possible approaches to prevention and intervention needs. ZEFQ. https://doi.org/10. 1016/j.zefq.2017.06.005

43. Wendsche J, Ghadiri A, Bengsch A et al (2017) Antecedents and outcomes of nurses' rest break organization: a scoping review. Int J Nurs Stud 75:65-80

44. Wendsche J, Hacker W, Wegge J (2017) Understaffing and registered nurses' turnover: the moderating role of regular rest breaks. Ger J Hum Resour Manage 31:238-259

45. Wirth T, Kozak A, Schedlbauer G et al (2016) Health behaviour, health status and occupational prospects of apprentice nurses and kindergarten teachers in Germany: a cross-sectional study. J Occup Med Toxicol. https://doi.org/10.1186/ s12995-016-0116-7

46. World Health Organization (2020) Global strategy on diet, physical activity and health. Physical activity. https://www.who.int/dietphysicalactivity/pa/ en/.Zugegriffen: 18. Mai 2020

47. Zapka JM, Lemon SC, Magner RP et al (2009) Lifestyle behaviours and weight among hospitalbased nurses. J Nurs Manag 17:853-860

48. Zeiher J, Kuntz B, Lange C (2017) Rauchen bei Erwachsenen in Deutschland. J Health Monit 2:59-65 